\title{
An Autopsy Case of Isolated Eosinophilic Coronary Periarteritis: A Limited Form of Churg-Strauss Syndrome or a New Entity?
}

\author{
Kayano TAIRA, Rikiya TsunodA*, Tatsuya Watanabe, Akihisa Fujino, \\ Astuhiro OGYU and Kouichi AsHIKAwA
}

\begin{abstract}
A 52-year-old man without a history of asthma or allergic diseases died of ventricular fibrillation early in the morning. His autopsy revealed no significant findings, except for a mild mural-thickening localized at the proximal region of the right coronary artery. Microscopic examination showed periarteritis with infiltration of numerous eosinophils in the adventitia. No significant vasculitis was found in any other organs. Based on the findings this seems to be the second reported case of isolated eosinophilic coronary periarteritis. (Internal Medicine 44: 586-589, 2005)
\end{abstract}

Key words: vasospastic angina, sudden death, eosinophilic periarteritis, coronary, Churg-Strauss syndrome

\section{Introduction}

Coronary periarteritis with eosinophil infiltration has been observed in hypereosinophilic syndrome or systemic vasculitis such as Churg-Strauss syndrome (CSS) and Wegener's granulomatosis. We report an autopsy case of sudden cardiac death because of vasospastic angina with isolated eosinophilic coronary periarteritis. This is the second reported case found in the literature.

\section{Case report}

A 48-year-old man was admitted to our hospital in September 1998. He had complained of chest discomfort in the early morning during the preceding 5 months. He had no history of hypertension bronchial asthma, paranasal sinusitis, drug allergy or neuropathy. He had a history of cigarette smoking (20 cigarettes per day for 28 years). His father died of unknown acute heart failure at 59 years of age and his mother had well-controlled sick sinus syndrome with pacemaker implantation. On admission, his blood pressure was $118 / 78 \mathrm{mmHg}$, pulse rate was $72 / \mathrm{min}$ and body temperature was $36.4^{\circ} \mathrm{C}$. His electrocardiogram (ECG) was normal, with no ST-segment elevation or depression. Chest X-ray showed no abnormality in the lung field. The cardiothoracic ratio (CTR) was $44.2 \%$. The hematological data showed white blood cells (WBC) 9,400/ $\mu 1$ (in hemography: neutrophil rate $78.2 \%$, eosinophil rate $0.8 \%$, basophil rate $0.1 \%$, and lymphocyte rate $18.5 \%$ ), and red blood cells (RBC) $471 \times 10^{4} / \mu 1$. Erythrocyte sedimentation rate was $1 \mathrm{~mm} / 1$ hour and $\gamma$ globulin was $0.99 \mathrm{~g} / \mathrm{dl}$. Blood chemical values and laboratory test results were normal, except that hepatitis B surface antigen was $(+)$, hepatitis $C$ virus antibody $(-)$, and $C$ reactive protein $0.0 \mathrm{mg} / \mathrm{dl}$. Hepatitis B surface antibody, hepatitis $\mathrm{B}$ core antigen and antibody, and hepatitis B envelope antigen and antibody were not examined. Coronary angiography did not show any organic stenosis in the right or left coronary arteries, but spontaneous spasm of 50\% was observed in seg. 2 of the right coronary artery. Ergonovine malate (0.4 $\mathrm{mg}$ ) intracoronary administration evoked diffuse spasm in the left anterior descending artery (LAD) and in the left circumflex artery (LCX). The spasm was released after intracoronary administration of nitroglycerin. Based on these findings, we diagnosed the patient with vasospastic angina. After medication with calcium antagonist and nicorandil, the anginal attack was improved. However, the patient decided to stop medication after one year, and used sublingual administration of nitroglycerin against anginal attack.

On June 6, 2002, at the age of 52 years, a severe anginal attack awoke him at 4:50 AM. The sublingual nitroglycerin was not effective and he gradually lost consciousness. When

From the Department of Cardiovascular Medicine and *the Department of Pathology, Yonezawa City Hospital, Yonezawa

Received for publication July 27, 2004; Accepted for publication December 24, 2004

Reprint requests should be addressed to Dr. Kayano Taira, the Department of Cardiovascular Medicine, Yonezawa City Hospital, 6-36 Aioi-cho Yonezawa, Yamagata 992-0085 


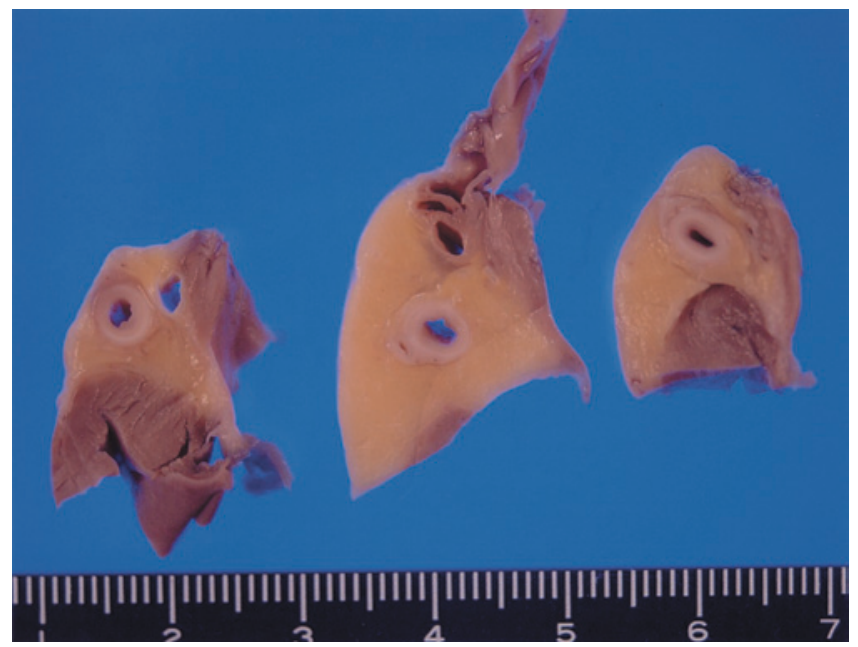

Figure 1. Cross-section of a proximal segment of the right coronary artery. Fibrous thickening was found in the intima, causing approximately $\mathbf{5 0 \%}$ luminal stenosis.

emergency medical services monitored his ambulatory ECG, ventricular fibrillation was recognized. He received cardioversion 4 times before arrival at our hospital. On admission, his respiratory rate was 1-2 times per minute, and the ECG monitor indicated cardiac arrest. Chest X-ray showed slight congestion and a CTR of 56\%. The results of laboratory examination showed WBC $15,900 / \mu \mathrm{l}, \mathrm{RBC} 446 \times 10^{4} / \mu \mathrm{l}$, aspartate transaminase (AST) $271 \mathrm{IU} / l$, alanine transaminase (ALT) $139 \mathrm{IU} / l$, lactate dehydrogenase (LDH) $992 \mathrm{IU} / l$, creatine kinase (CPK) 1,368 IU/l (CK-MB 274.8 IU/l), and CRP $0.0 \mathrm{mg} / \mathrm{dl}$. Myeloperoxidase-antineutrophil cytoplasmic antibody (MPO-ANCA) was not examined. We performed ventricular pacing and cardiopulmonary resuscitation, but he died at 8:45 AM on the same day. Post-mortem CT scan did not detected any significant intracranial abnormality.

\section{Macroscopic and histological findings}

The patient was autopsied one and a half hours after death. Both lungs showed marked passive congestive pulmonary edema (1,046 g: $758 \mathrm{~g})$ with smoking-induced pleural anthracosis. The heart weighed $378 \mathrm{~g}$ with concentric hypertrophy of the left ventricle. There was no athero- or arteriosclerosis detected in the vascular system. However, a segment of the right coronary artery approximately $10 \mathrm{~mm}$ in length $5 \mathrm{~mm}$ distal from the beginning was extraordinarily elastic-hard, showing up to $50 \%$ obstructive transmural grayish-white thickening on cross section (Fig. 1). Microscopic examination of this vascular lesion demonstrated 1.5 to 2 times the normal intimal fibrous thickening (Fig. 2). The endothelial cells were well preserved, however, a few eosinophils had infiltrated under the subendothelial layer. Specific staining did not show any damage to the elastic fibers of the media or fibrinoid necrosis, suggesting that there was no evidence of vasculitis. It is a specific finding in this case that

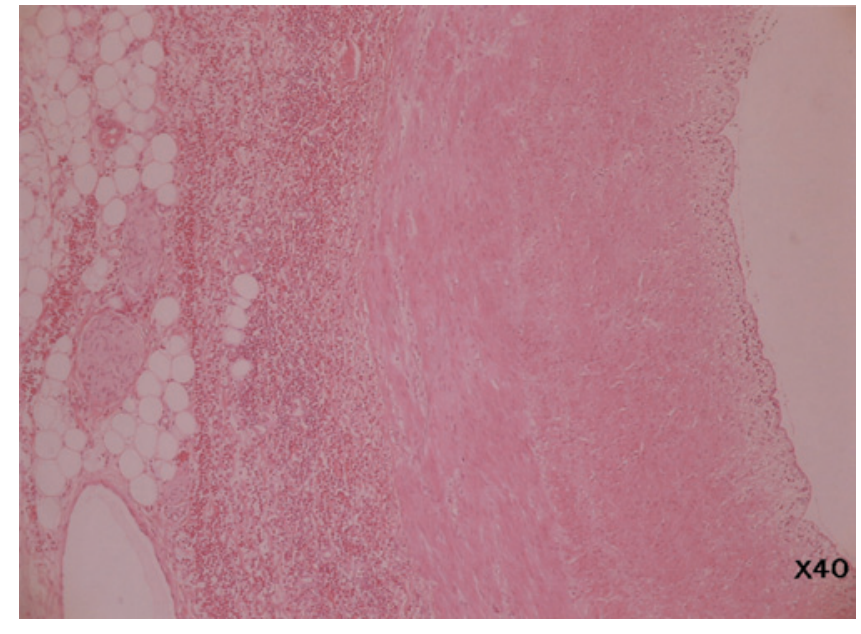

Figure 2. Inflammatory infiltration was localized just within the adventitial and periadventitial regions of the right coronary artery. Numerous eosinophils were diffuse. A few eosinophils were also scattered in the subendothelial layer. The intima showed 1.5-2 times normal thickening with fibrosis. Fibrinoid necrosis was not detected in any section (HE stain, $\times 40$ ).

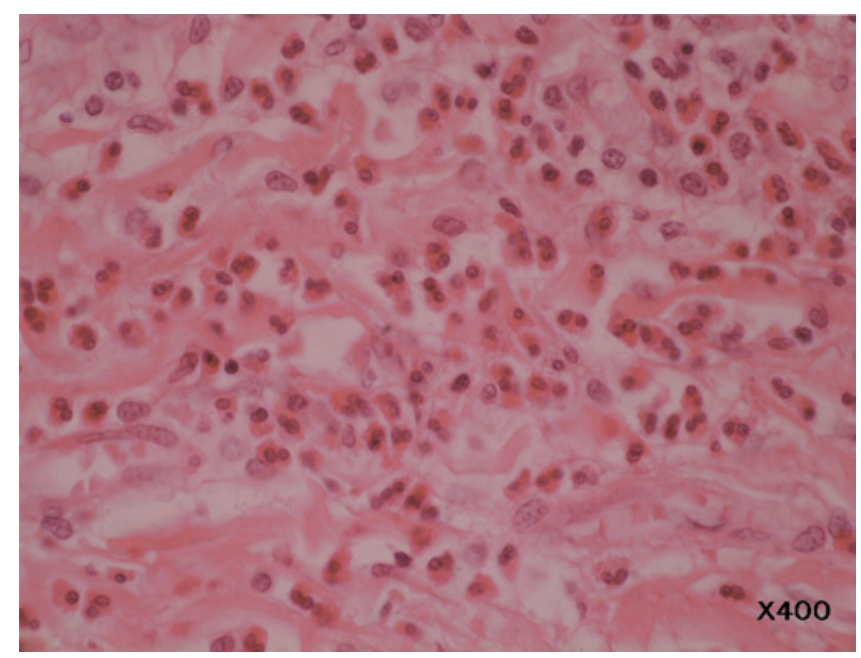

Figure 3. Eosinophil infiltration in the adventitia with a few $\mathrm{T}$ lymphocytes (CD4>CD8) and plasma cells (HE stain, $\times 400)$.

numerous eosinophils diffusely infiltrated in the periadventitial and adventitial layers (Fig. 3), with some scattered $\mathrm{T}$ cells (CD4>CD8) and plasma cells. No chronic specific granuloma or necrotic inflammation was detected. The eosinophilic periarteritis described above was not found in any other coronary segments or other arteries in any of the organs investigated. Many small foci of marked contractionband myocardial necrosis were randomly scattered throughout both ventricles. There was no significant eosinophil infiltration in the interstitial tissues of the heart, spleen or any other organs. 


\section{Discussion}

We presented a case report of sudden cardiac death due to vasospastic angina. Pathologically, there was arteritis in the right coronary artery with intimal fibrous thickening that induced $50 \%$ stenosis of the lumen. Remarkable infiltration of eosinophils was localized in the adventitia, but the medial smooth muscle layer was well preserved. This case resembles Churg-Strauss syndrome (CSS) (1) in that vasculitis was found in medium-sized arteries, and showed eosinophilic infiltration.

The classification criteria for Churg-Strauss syndrome (CSS) of the American College of Rheumatology (1990) (2) include the following: 1) bronchial asthma 2) eosinophilia (more than $10 \%$ of WBC) 3) mono- or polyneuropathy 4) non-fixed pulmonary infiltrates 5) paranasal sinus abnormality and 6) extravascular eosinophil infiltrates. These criteria were selected to discriminate CSS from other forms of vasculitis.

There have been some reports of a limited form of ChurgStrauss syndrome with isolated eosinophilic coronary periarteritis. In 1989, Lie and Bayardo (3) reported the sudden death of a 39-year-old man with chronic asthma. In their case, marked eosinophils existed in the adventitia and outer media of the coronary arteries, and isolated necrotizing eosinophilic myocarditis was scattered throughout the subendcardial region of the left ventricle. Tissue eosinophilia was also prominent in the spleen. In 1992, Hunsaker et al (4) described a case of coronary arterial dissection with eosinophilic periarteritis and fibrinoid necrosis in a 57-year-old woman, who also had angina for several months before death. She had a history of allergies to numerous agents and food. In that case, there was no finding of eosinophilia in the peripheral blood, however, she had allergic disease, and extravascular eosinophils were found in the myocardium. A limited form of CSS demonstrates eosinophilic vasculitis and extravascular granuloma in single organs or tissues without evidence of systemic disease (5). Therefore, these two cases are highly suggestive of a limited form of CSS. In comparison with these two cases, the present case had no findings of extravascular granulomas or extravascular tissue infiltration by eosinophils. Additionally, he had no history of allergic disease. Asthma is a central feature of CSS (6). These clinical and histopathological finding strongly indicate that our case did not have this limited form of CSS.

In 1989, Kajihara et al (7) reported a similar case as a new entity of vasculitis in a 40-year-old man who died after hospitalization for unstable angina pectoris. He had no history of allergic history, and eosinophil infiltration in the adventitia and fibrous thickening of the intima in the left anterior descending and right coronary arteries were observed, as in our case. Virmani et al (8) explained that eosinophilic periarteritis is distinguished from CSS angitis by the absence of necrosis and soft tissue granulomas. The infiltration of eosonophils is entirely adventitial, and the media of the artery is intact with a patent lumen. Thus, these two similar cases differ from the previous two cases of CSS, and are suggested to be a new entity of isolated eosinophilic coronary periarteritis.

Recently, hepatitis B virus infection has been observed to be associated with polyarteritis nodosa or autoimmune reactions (9). Although we did not examine the circulating immuno-complexes and cryoglobulins, in this case, there was only monoarteritis in the coronary artery, and no pathological or clinical sign of polyarteritis nodosa.

It has been previously reported that spasm of the human coronary artery is associated with adventitial inflammation (10). Shimokawa (11) suggested that under long-term stimulation of the adventitia with some inflammatory cytokines, smooth muscle phenotypes are altered, resulting in a coronary vasospastic response and slight neointimal formation. Platelet-activating factor (PAF) is a lipid mediator, released from activated leukocytes and eosinophils. Kozai et al (12) reported that in inflammatory coronary lesions, PAF causes hyperconstriction in vivo. These studies showed that longterm treatment with inflammatory cytokines and mediators causes intimal hypertrophy and vasospastic responses. Leukotriene C4 (LTC4), a class of biologically active lipids acting on smooth muscles from the bronchi and intestine, is synthesized and released from leukocytes and eosinophils. Komohara et al (13) reported that human coronary smooth muscle cells are stimulated with LTC4 by increasing the intracellular calcium concentration. This action was expected to induce contraction of coronary smooth muscle.

In conclusion, we report a case of isolated eosinophilic coronary periarteritis, as a new entity distinct from CSS. We emphasize that this type of coronary periarteritis causes cardiac sudden death as a consequence of vasospastic angina. The marked infiltration of eosinophils in the adventitia causes intimal thickening and vasospasm of the coronary artery through cytokines and lipid mediators.

Acknowledgements: We are grateful to Dr. Y. Sakomura for valuable advice.

\section{References}

1) Churg J, Strauss L. Allergic granulomatosis, allergic angiitis and polyarteritis nodosa. Am J Pathol 27: 277-301, 1951.

2) Masi AT, Hunder GG, Lie JT, et al. The American College of Rheumatology 1990 criteria for the classification of Churg-Strauss syndrome. Arthritis Rheum 33: 1094-1100, 1990.

3) Lie JT, Bayardo RJ. Isolated eosinophilic coronary arteritis and eosinophilic myocarditis: A limited form of Churg-Strauss syndrome. Arch Pathol Lab Med 113: 199-201, 1989.

4) Hunsaker JC 3rd, O'Connor WN, Lie JT. Spontaneous coronary arterial dissection and isolated eosinophilic coronary arteritis: Sudden cardiac death in a patient with a limited variant of Churg-Strauss syndrome. Mayo Clin Proc 67: 761-766, 1992.

5) Lie JT. Limited forms of Churg-Strauss syndrome. Pathol Annu 28 : 199-220, 1993.

6) Lanham JG, Elkon KB, Pusey CD, Hughes GR. Systemic vasculitis with asthma and eosinophilia: a clinical approach to the Churg-Strauss Syndrome. Medicine 63: 65-81, 1984.

7) Kajihara H, Kato Y, Takanashi A, et al. Periarteritis of coronary 


\section{Isolated Eosinophilic Coronary Periarteritis}

arteries with severe eosinophilic infiltration. A new pathologic entity (eosinophilic periarteritis)? Pathol Res Pract 184: 46-52, 1989.

8) Virmani R, Atkinson JB, et al. Churg-Strauss angitis and eosinophilic periarteritis. in: Cardiovascular Pathology (Major problems in pathology, Vol. 40), 2001: 129-134.

9) Trepo C, Guillevin L. Polyarteritis nodosa and extrahepatic manifestations of HBV infection: the case against autoimmune intervention in pathogenesis. J Autoimmun 16: 269-274, 2001.

10) Kohchi K, Takebayashi S, Hiroki T, Nobuyoshi M. Significance of adventitial inflammation of the coronary artery in patients with unsta- ble angina: results at autopsy. Circulation 71: 709-716, 1985.

11) Shimokawa H. Cellular and molecular mechanisms of coronary artery spasm. Lessons from animal models. Jpn Circ J 64: 1-12, 2000.

12) Kozai $T$, Shimokawa $H$, Yamawaki $T$, et al. Platelet activating factor causes hyperconstriction at the inflammatory coronary lesions in pigs in vivo. Coron Artery Dis 8: 423-432, 1997.

13) Komohara M, Takasaki J, Matsumoto M, et al. Functional characterization of cysteinyl leukotriene CysLT2 receptor on human coronary artery smooth muscle cells. Biochem Biophys Res Commun 287: 10881092, 2001 